\title{
Quantization of Masses of Elementary Particles with Micrononcausal Structures.
}

\section{H. Enatsu}

Department of Physics, Ritsumeikan University - Kyoto, 603 Japan

(Nuovo Cimento $A, 95,269(1986)$ )

The following corrections should be noted in this paper:

p. 271, line 2:

p. 272 , line 6 from bottom: the last line:

p. 278 , line 2 :

p. 279 , line 4 after eq. (5.6):

p. 284, line 3 after eq. (5.38):

p. 285, line 1 :

p. 286, line 1 :

p. 287 , eq. $(6.10)$ :

eq. (6.13): a systems of $\rightarrow$ a system of

Holster $\rightarrow$ Hostler

in ref. ${ }^{(1)} \rightarrow$ in I

in ref. ${ }^{(1)} \rightarrow$ in $I$

$m_{4} \rightarrow M_{4}$

$V_{1}(x)+V(x) \rightarrow V_{1}^{L}(x)+\frac{1}{2} V_{2}(x)$

Heusenberg $\rightarrow$ Heisenberg

the $S$-matrix is $(6.3) \rightarrow$ the $S$-matrix is

$\alpha_{\mu \nu} \rightarrow d_{\mu \nu}$

$\left[\begin{array}{c}K_{1 \nu}(M R) \\ M R\end{array}+\delta_{\mu v} \frac{K_{2}(M R)}{R^{2}}-M Z_{\mu} Z_{v} \frac{K_{3}(M R)}{R^{2}}\right]$

$\rightarrow\left[\delta_{\mu \nu} \frac{K_{1}(M R)}{M R}+\delta_{\mu \nu} \frac{K_{2}(M R)}{M^{2} R^{2}}-Z_{\mu} Z_{v} \frac{K_{3}(M R)}{M R^{3}}\right]$

$\sin \varphi \rightarrow \sin \varphi^{\prime}$

$S_{1 c} \rightarrow S_{2 c}$

$\left[1-2 \varrho \cos \beta+\varrho^{2}\right]^{-2} \rightarrow\left[1-2 \varrho \cos \beta+\varrho^{2}\right]^{-3}$

$Y \rightarrow \varrho$

$\left(\mathrm{d}^{4} x_{\mu}\right)_{E} \rightarrow\left(\mathrm{d}^{4} x\right)_{E}$

$m^{2}, \rightarrow m_{s}^{2}, \quad M^{2} \rightarrow M_{V}^{2}$. 